\title{
Direct Modulation of Widely Tunable Twin-Guide Lasers
}

\author{
Reinhard Laroy, René Todt, Ralf Meyer, Markus-Christian Amann, Senior Member, IEEE, \\ Geert Morthier, Senior Member, IEEE, and Roel Baets, Senior Member, IEEE
}

\begin{abstract}
The possibility to directly modulate widely tunable lasers up to several gigahertz is desirable in telecom applications. We discuss the dynamic properties of the recently proposed widely tunable twin-guide laser concept. It has promising prospects with a maximum theoretical bandwidth above $20 \mathrm{GHz}$ and the 3-dB bandwidth at $250 \mathrm{~mA}$ indicates that an actual bandwidth of $12 \mathrm{GHz}$ should be possible. The current lasers were not designed for highspeed modulation, so only 1-GHz modulation can be reached at the moment.
\end{abstract}

Index Terms-Modulation, semiconductor laser, tunable laser.

\section{INTRODUCTION}

W IDELY tunable semiconductor laser diodes with tuning ranges of several tens of nanometers are considered key components in optical telecommunication networks and sensor applications.

At present, several widely tunable laser concepts have been presented and many of them are distributed Bragg reflector laser diodes with one or two passive reflector sections [1]-[3]. However, also widely tunable distributed feedback lasers [4]-[6] have received attention in recent years. They only require two control currents, so they are easier to characterize and to control.

One of these devices is the sampled grating tunable twinguide (SG-TTG) laser [4]. This new concept is based on the tunable twin-guide (TTG) laser concept [7] and uses the Verniereffect to obtain a large tuning range. Electronic tuning over 40-nm wavelength range has recently been demonstrated for these devices [8].

In this letter, the dynamic properties of these SG-TTG lasers will be examined. The possibility to directly modulate the laser up to several or even $10 \mathrm{GHz}$ is desirable for low-cost digital and analog optical telecommunication applications (e.g., in optical access networks).

We will first report on relative intensity noise (RIN) measurements that have been performed to obtain the intrinsic modulation bandwidths. A theoretical maximum modulation bandwidth has been extracted out of these measurements. In a second part,

Manuscript received January 26, 2006; revised March 29, 2006. This work was supported by the European Union under the Information Society Technologies (IST) Project IST-2000-28244 NEWTON. The work of R. Laroy was supported by the Institute for the Promotion of Innovation by Science and Technology in Flanders (IWT) under a specialization grant.

R. Laroy, G. Morthier, and R. Baets are with the Department of Information Technology, IMEC/Ghent University, B-9000 Gent, Belgium.

R. Todt, R. Meyer, and M.-C. Amann are with the Walter Schottky Institute, Technische Universität München, D-85748 Garching, Germany.

Digital Object Identifier 10.1109/LPT.2006.876752
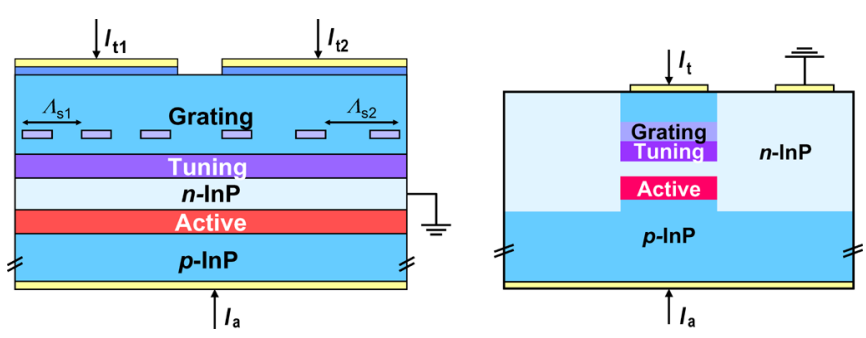

Fig. 1. Schematic drawing of a widely tunable twin guide laser with sampled gratings. (Color version available online at http://ieeexplore.ieee.org.)

we will briefly discuss the first experiments with directly modulated laser diodes.

\section{Device Structure}

A widely TTG laser [4] is a two section TTG laser [7], in which both sections contain a sampled grating or a superstructure grating with different superperiod. The device structure of a TTG laser (Fig. 1) consists of two p-n junctions with an n-separation layer in the middle that electronically decouples the active layer and tuning layer, so gain and filtering can be controlled independently.

Current injection into the tuning layer is used to change the effective refractive index and the Vernier effect creates tuning over a wide wavelength range (several tens of nanometers). Only two tuning currents are required to obtain full wavelength coverage, which makes the characterization substantially less time consuming. A high output power and a short device length are possible.

The SG-TTG laser has a competitive device performance. Recently [8], SG-TTG laser diodes with a tuning range of more than $40 \mathrm{~nm}$ and output powers higher than $10 \mathrm{~mW}$ were reported (Fig. 2). The slope is around $0,075 \mathrm{~mW} / \mathrm{mA}$ and decreases due to leakage currents to $0.015 \mathrm{~mW} / \mathrm{mA}$ around $250 \mathrm{~mA}$.

For an untuned laser, the output frequency is $192.4 \mathrm{THz}$ and the threshold is reached at an active region current of $27 \mathrm{~mA}$, corresponding with a threshold current density of $1.7 \mathrm{kA} / \mathrm{cm}^{2}$. Due to carrier-induced losses, this threshold current density goes up to $6 \mathrm{kA} / \mathrm{cm}^{2}$.

\section{NOISE MEASUREMENTS}

The theoretical maximum modulation frequency under small signal modulation can be derived from the measurements of the RIN. The RIN describes the amplitude fluctuations in the optical field due to spontaneous emission. 


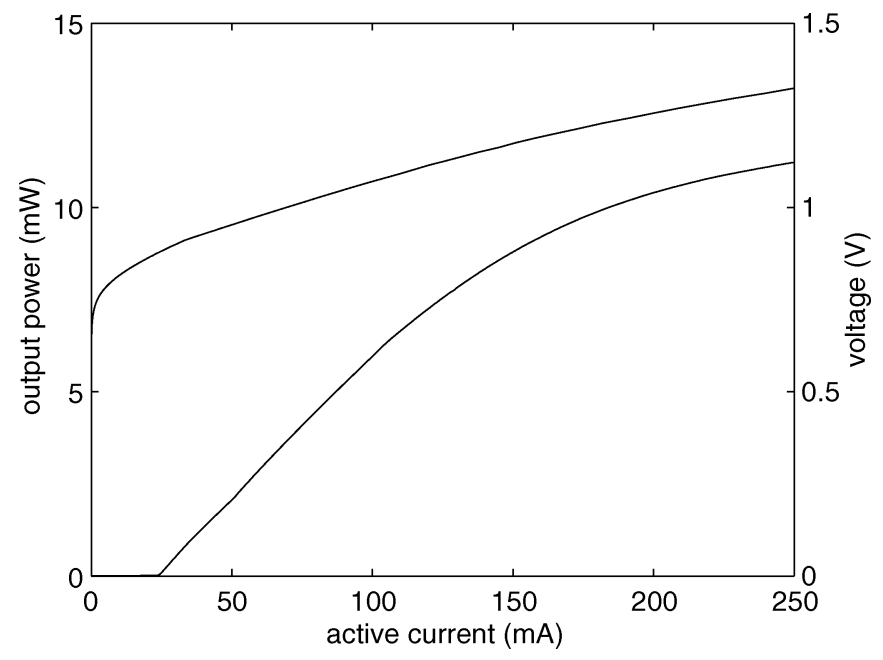

Fig. 2. Light output-current-voltage characteristic of a SG-TTG.

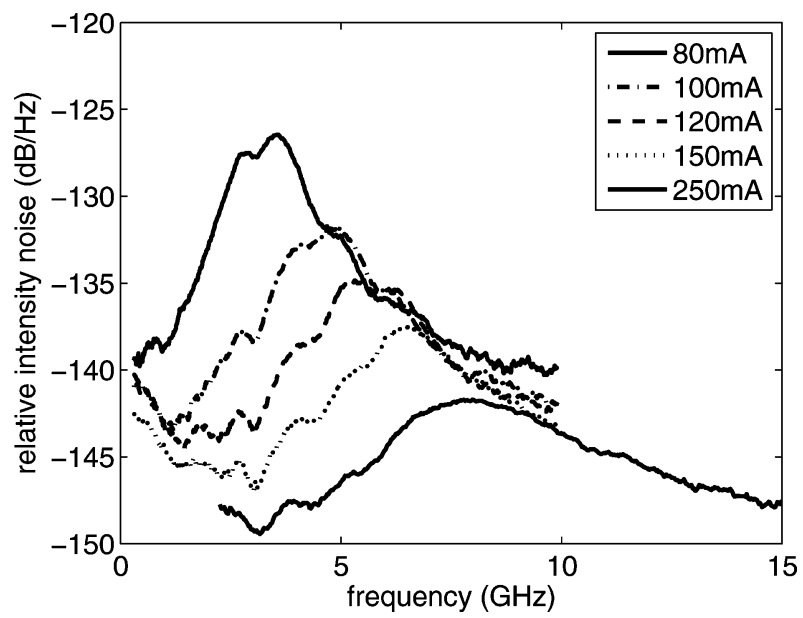

Fig. 3. RIN measurements of an SG-TTG laser for different active current values and tuning currents set to an output frequency of $195.1 \mathrm{THz}$.

The measured RIN results of an SG-TTG laser with a total length of $1200 \mu \mathrm{m}$ for different values of the active current and zero tuning currents are shown in Fig. 3. These measurements can be fitted to the formula for the RIN to extract the resonance frequency $f_{r}$ and the damping $\gamma$

$$
\operatorname{RIN}(f)=A \frac{f^{2}+\left(\frac{\gamma}{2 \pi}\right)^{2}}{\left(f_{r}^{2}-f^{2}\right)^{2}+\left(\frac{\gamma}{2 \pi}\right)^{2} f^{2}} .
$$

Far above threshold, the resonance frequency $f_{r}$ and the damping $\gamma$ are connected by the $K$-factor [9]

$$
\gamma=\gamma_{0}+K f_{r}^{2}
$$

The $K$-factor is determined by the photon lifetime of the laser cavity and is inversely proportional to the maximum intrinsic modulation bandwidth [9]

$$
f_{\text {max }, \text { damping }}=\frac{2 \pi \sqrt{2}}{K} .
$$

The extracted theoretical values for the maximum intrinsic modulation bandwidth are given for several ITU frequencies in

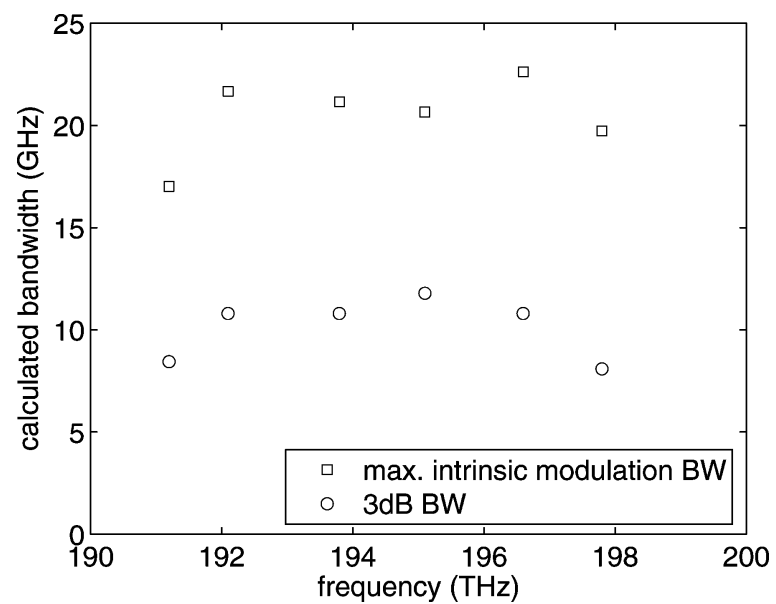

Fig. 4. Theoretical indication of actual bandwidth of an SG-TTG laser through modulation response formula.

Fig. 4. The variation of this theoretical bandwidth between 17 and $23 \mathrm{GHz}$ is due to the wavelength dependence of the material and optical cavity parameters. These values for $f_{\max \text {,damping }}$ are, however, the highest reported so far for tunable laser diodes. Only the Modulated Grating Y laser comes in the neighborhood [3] with a theoretical maximum intrinsic modulation bandwidth up to $20 \mathrm{GHz}$.

In practice, the performance of the device will be worse than predicted due to the presence of parasitics like stray capacitances parallel to the active region, the resistance of the semiconductor layers, the inductance of bond wires, etc.

The same parameters from the formula (1) are used in the small-signal modulation response formula

$$
|M(f)|^{2}=\frac{1}{1+\left(\frac{f}{f_{p}}\right)^{2}} \frac{f_{r}^{4}}{\left(f_{r}^{2}-f^{2}\right)^{2}+\left(\frac{\gamma}{2 \pi}\right)^{2} f^{2}}
$$

with $f_{p}$ the parasitic cutoff frequency. An indication of the a more realistic theoretical 3-dB bandwidth (without parasitics) can be obtained by inserting the extracted parameters from our RIN fitting at an active current of $250 \mathrm{~mA}$ in this modulation response neglecting the parasitic factor. This leads to the $3-\mathrm{dB}$ bandwidth values shown in Fig. 4. For all measured ITU frequencies, a bandwidth higher than $8 \mathrm{GHz}$ and up to $12 \mathrm{GHz}$ is obtained.

The extracted bandwidth is quite uniform over the whole tuning range, this is due to two counteracting effects. The tuning increases the threshold current considerably and this has a decreasing effect on the resonance frequency. On the other hand, the spectral variation of $d g / d N$ (Fig. 5) compensates this because tuning is toward the high-frequency side where $d g / d N$ is larger.

These two counteracting effects result in a more or less uniform bandwidth throughout most of the tuning range and, simultaneously explain for the decrease of the bandwidth at the edges of the tuning range where the $I-I_{\text {th }}$ decrease is the largest and the $d g / d N$ variations are not strong enough anymore to compensate this. By adjusting the detuning of the gain peak and moving the grating center wavelength to lower frequencies, the average $d g / d N$ value can be increased without 


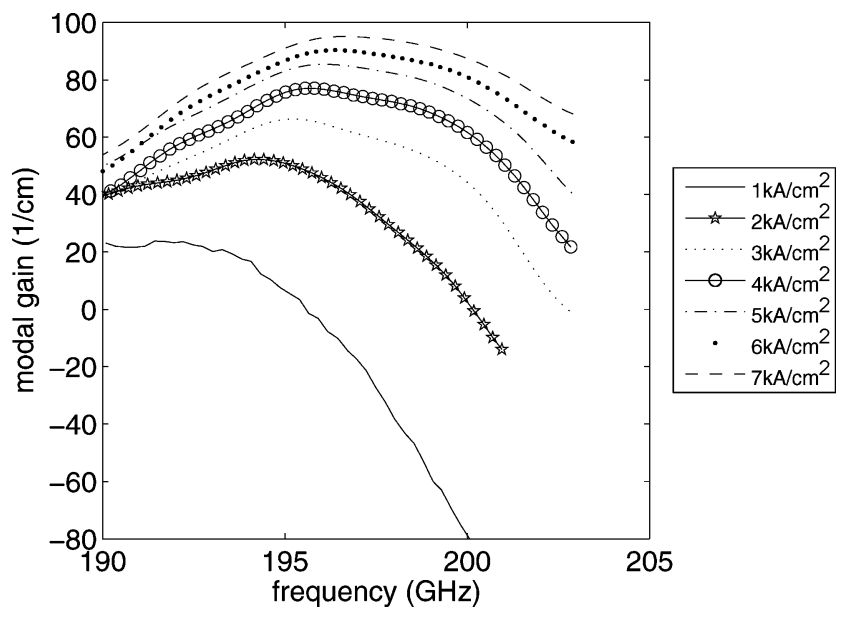

Fig. 5. Modal gain changes due to frequency and current density variations.

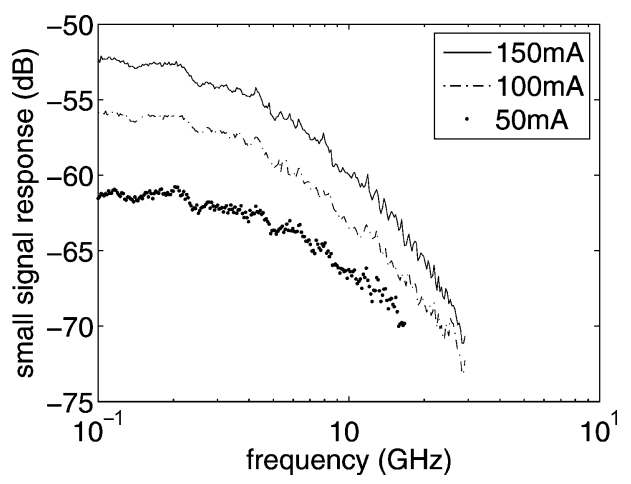

Fig. 6. Small-signal intensity modulation of a TTG laser at different active currents.

decreasing $I-I_{\text {th }}$ and, therefore, the bandwidth can on average be increased.

Additionally, modified concepts have been presented [10], [11] to increase the output power or the pumping efficiency of the TTG lasers considerably and, thereby, probably also the modulation bandwidth.

\section{SMall-Signal Modulation}

The SG-TTG laser sample has been probed with a Picoprobe $40 \mathrm{~A}$, a high-frequency needle for small-signal modulation up to $40 \mathrm{GHz}$. A bias-T combines the dc and ac signal and isolates the sources from each other. The output signal is detected by a highspeed photodiode and processed by an HP71400 light wave signal analyzer to extract the amplitude of the optical power modulation.

The small-signal behavior for an SG-TTG laser at different active currents is shown in Fig. 6. A 3-dB cutoff frequency is reached around $1 \mathrm{GHz}$. The dynamic properties remain unchanged when the active current is increased (Fig. 6) or when the laser is tuned, indicating that presently the parasitic effects dominate the dynamic behavior.
The low modulation bandwidth is not a surprise because the present batch of devices was not designed for high frequency operation. Voltage-current measurements (Fig. 2) show that the parastic series resistance is around $5 \Omega$, so the parasitic capacitance is around $32 \mathrm{pF}$ for a $1-\mathrm{GHz} 3-\mathrm{dB}$ cutoff frequency. The huge contact pad $\left(1200 \times 110 \mu \mathrm{m}^{2}\right)$ contributes to $8 \mathrm{pF}$ of this $32-\mathrm{pF}$ capacitance and can be almost completely eliminated simply by reducing its size and using a more suitable passivation like BCB. The remaining 24-pF capacity is most likely caused by the large p-n junction with a width of $25 \mu \mathrm{m}$. To get close to the magical $10 \mathrm{GHz}$, a capacity of $3.2 \mathrm{pF}$ is needed (assuming again a $5-\Omega$ series resistance). This can be obtained with a $p-n$ junction of about $5-\mu \mathrm{m}$ width. This is feasible with some design optimizations.

\section{CONCLUSION}

The widely TTG laser has promising prospects. It has a maximum theoretical bandwidth above $20 \mathrm{GHz}$ and the $3-\mathrm{dB}$ bandwidth at $250 \mathrm{~mA}$ indicates that an actual bandwidth of $12 \mathrm{GHz}$ is possible when the parasitics can be minimized sufficiently. The current lasers were not designed for high-speed modulation, so only $1-\mathrm{GHz}$ modulation can be reached at the moment.

\section{REFERENCES}

[1] A. Saavedra, P.-J. Rigole, E. Goobar, R. Schatz, and S. Nilsson, "Relative intensity noise and linewidth measurements of a widely tunable GCSR laser," IEEE Photon. Technol. Lett., vol. 10, no. 4, pp. 481-483, Apr. 1998.

[2] M. Majewski, J. Barton, L. Coldren, Y. Akulova, and M. Larson, "Direct intensity modulation in sampled-grating DBR lasers," IEEE Photon. Technol. Lett., vol. 14, no. 6, pp. 747-749, Jun. 2002.

[3] M. Chacinski, M. Isaksson, and R. Schatz, "High-speed direct modulation of widely tunable MG-Y laser," IEEE Photon. Technol. Lett., vol. 17, no. 6, pp. 1157-1159, Jun. 2005.

[4] G. Morthier, B. Moeyersoon, and R. Baets, "A $\lambda / 4$-shifted sampled or superstructure grating widely tunable twin-guide laser," IEEE Photon. Technol. Lett., vol. 13, no. 10, pp. 1052-1054, Oct. 2001.

[5] H. Ishii, Y. Kondo, F. Kano, and Y. Yoshikuni, "A tunable distributed amplification DFB laser diode (TDA-DFB-LD)," IEEE Photon. Technol. Lett., vol. 10, no. 1, pp. 30-32, Jan. 1998.

[6] S. Kim, Y. Chung, S.-H. Oh, and M.-H. Park, "Design and analysis of widely tunable sampled grating DFB laser diode integrated with sampled grating distributed Bragg reflector," IEEE Photon. Technol. Lett., vol. 16, no. 1, pp. 15-17, Jan. 2004.

[7] M.-C. Amann, S. Illek, C. Shanen, and W. Thulke, "Tuning range and threshold current of the tunable twin-guide (TTG) laser," Electron. Lett., vol. 1, pp. 253-254, Feb. 1989.

[8] R. Todt, T. Jacke, R. Meyer, J. Adler, R. Laroy, G. Morthier, and M.-C. Amann, "Sampled grating tunable twin-guide laser diodes with over 40-nm electronic tuning range," IEEE Photon. Technol. Lett., vol. 17, no. 12 , pp. 2514-2516, Dec. 2005.

[9] L. A. Coldren and S. W. Corzine, Diode Lasers and Photonic Integrated Circuits. New York: Wiley, 1995.

[10] B. Schmidt, S. Utek, R. Gessner, and M.-C. Amann, "Design and realization of a buried-heterostructure tunable-twin-guide laser diode with electrical blocking regions," IEEE J. Quantum Electron., vol. 35, no. 5, pp. 794-802, May 1999.

[11] R. Todt, T. Jacke, R. Meyer, and M.-C. Amann, "High output power tunable twin-guide laser diodes with improved lateral current injection structure," Electron. Lett., vol. 41, pp. 190-191, Apr. 2005. 